\title{
Predicting Preeclampsia with Noninvasive Measures of Endothelial Dysfunction: A Pilot Study
}

\author{
Emmanuel U. Emeasoba, MD ${ }^{1}$ Rodney A. McLaren Jr., MD ${ }^{2}$ Rebecca Landau, BA ${ }^{1}$ \\ Jeremy Weedon, PhD, MA, BS ${ }^{3}$ Shoshana Haberman, MD, PhD ${ }^{2}$ Howard Minkoff, MD²,4 \\ Jacob Shani, MD ${ }^{1}$ Nadine Montemarano, $\mathrm{MD}^{1}$
}

${ }^{1}$ Department of Cardiology, Maimonides Medical Center, Heart \&

Address for correspondence Rodney A. McLaren, MD, Department of Vascular Institute, Brooklyn, New York

2 Department of Obstetrics and Gynecology, Maimonides Medical Obstetrics and Gynecology, Maimonides Medical Center, 967 48th Center, Brooklyn, New York

${ }^{3}$ Department of Epidemiology and Biostatistics, SUNY Downstate

Medical Center, Brooklyn, New York

${ }^{4}$ Department of Obstetrics and Gynecology, SUNY Downstate

Medical Center, Brooklyn, New York

Street, Brooklyn, NY 11219 (e-mail: rmclaren624@gmail.com).

Am J Perinatol Rep 2020;10:e20-e25.

\begin{abstract}
Objective This study evaluates the assessment of endothelial function and its prediction for preeclampsia among women with high-risk factors.

Study Design A prospective cohort study of 107 pregnant women at 20 weeks or greater gestation with risk factors for developing preeclampsia. Endothelial dysfunction was assessed using peripheral arterial tonometry by generating a reactive hyperemia index (RHI) score. An index score of $<1.67$ was defined as endothelial dysfunction. The primary outcome was preeclampsia. Logistic regression was used to predict preeclampsia from $\mathrm{RHI}$ scores, body mass index, gestational age at RHI evaluation, history of preeclampsia, history of pregestational diabetes mellitus, chronic hypertension, and fetal number. A receiver operating characteristic plot was constructed to predict preeclampsia from the RHI score.

\section{Keywords}

- endothelial dysfunction

- peripheral arterial tone

- preeclampsia

- reactive hyperemia index

Results Among 107 women, 99 had interpretable RHI scores. Among those with an abnormal RHI $(n=61), 17(28 \%)$ developed preeclampsia. Among women with a normal score $(n=38)$, six $(16 \%)$ developed preeclampsia $(p=0.166)$. After logistic regression, there was no significant association. A receiver operating characteristic plot also revealed no association between $\mathrm{RHI}$ score and preeclampsia.

Conclusion An abnormal RHI score using peripheral arterial tonometry indicating endothelial dysfunction was not predictive of developing preeclampsia in this cohort. Future studies are needed to further evaluate this relationship.
\end{abstract}

Preeclampsia is a pregnancy-specific hypertensive disorder with multisystem involvement that occurs after 20 weeks of gestation in previously normotensive pregnant women. ${ }^{1}$ Pregnant women with risk factors, such as obesity, history of chronic hypertension, history of preeclampsia, nulliparity, multiple gestation, and diabetes, are considered to be at higher risk for developing preeclampsia. ${ }^{2}$ Additionally, women affected by preeclampsia have been shown to be at an increased risk for cardiovascular issues later in life, possibly due to the persistence of endothelial dysfunction. ${ }^{3-5}$ Previous studies have shown that preeclampsia is associated with arterial dysfunction-particularly increased arterial stiffness-during pregnancy, which may received

May 24, 2019

accepted after revision

October 15, 2019
DOI https://doi.org/

10.1055/s-0039-3401806. ISSN 2157-6998.
Copyright $\odot 2020$ by Thieme Medical

Publishers, Inc., 333 Seventh Avenue, New York, NY 10001, USA. Tel: +1(212) 760-0888
License terms

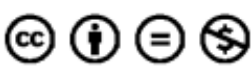


also be responsible for the increased risk of long-term cardiovascular complications. ${ }^{4-6}$ Whether endothelial dysfunction is causative or is merely an earlier manifestation of the disease process, it is possible that early markers of endothelial dysfunction can serve as predictors of preeclampsia during pregnancies, especially in women with preexisting risk factors.

Noninvasive techniques for assessing endothelial dysfunction have been described; however, many are user dependent and thus are not reliable. ${ }^{7}$ One of the most reproducible techniques to assess endothelial function is peripheral arterial tonometry. It is user independent and provides automatically calculated measurements of endothelial function. Although this method has been used extensively in nonpregnant patients, few studies have examined its utility in predicting preeclampsia. ${ }^{6,8,9}$ In one retrospective study, researchers measured postdelivery endothelial function of women who experienced preeclampsia to determine whether residual endothelial dysfunction may contribute to future cardiovascular issues, and found that there was persistent microcirculatory dysfunction in women with previous pregnancies complicated by preeclampsia. ${ }^{4,5}$ Whether this technique can be used to predict preeclampsia in women with high-risk factors, by detecting early signs of endothelial dysfunction, is currently not known. Our objective was to determine if there was a correlation between endothelial dysfunction determined by peripheral arterial tonometry and increased risk of developing preeclampsia in pregnant women.

\section{Methods}

We performed a prospective, single center, cohort study of pregnant women at risk for preeclampsia. After approval by the institutional review board at Maimonides Medical Center, a convenience sample of participants was recruited at the perinatal testing center of the department of obstetrics and gynecology. Inclusion criteria were 20 weeks of gestation or more with one of the following risk factors: advanced maternal age, preexisting hypertension, prepregnancy obesity (defined as body mass index of $30 \mathrm{~kg} / \mathrm{m}^{2}$ or greater), past personal and/or family history of preeclampsia, nulliparity, multiple pregnancy, pregestational and gestational diabetes, pregnancy by in vitro fertilization, and history of antiphospholipid syndrome. Women at gestational age of less than 20 weeks, women who had symptoms of preeclampsia, fetal distress, HELLP syndrome, eclampsia, or other complications requiring immediate intervention, were excluded from the study. ${ }^{4}$

Patients enrolled in the study had their blood pressure and heart rate measured, and had an endothelial function evaluation using the Endo-PAT 2000 device (Itamar Medical Ltd., Casarea, Israel). Blood pressure during perinatal visits was assessed using a standard, calibrated electronic sphygmomanometer. The device automatically inflated the cuff until over-systolic pressures have been reached and then deflated gradually, recording the blood pressure by means of oscillometric technology. During blood pressure measurements, the women were relaxed, seated, back supported, and legs uncrossed. Their arms were supported at the level of the heart, and blood pressure readings were taken after 5 minutes of quiet sitting. Afterward, in the same quiet room, the Endo-PAT test commenced.

\section{The Endo-PAT Test (Endothelial Function Assessment)}

Endothelial vasodilation function was assessed using the Endo-PAT 2000 device (Itamar Medical Ltd., Caesarea, Israel), which records endothelium-mediated changes in the digital pulse waveform known as the peripheral arterial tone (PAT) signal. After a brief resting period on a chair inclined at an angle of 45 degrees at room temperature, a blood pressure cuff was placed on the nondominant upper arm (experimental arm) while the other arm served as control. The subject's hands were placed on armchair support with the palm side down such that the fingers hung freely. A pair of plethysmographic probes was situated on the index finger of each hand such that they did not touch any other finger or object. These probes comprised a system of inflatable latex air cuffs connected by pneumatic tubes to the Endo-PAT device and controlled through a computer algorithm.

The probes were electronically inflated, and baseline pulse amplitude was measured from each fingertip for 5 minutes. Pulsatile volume changes in the distal digits induced pressure alterations in the finger probes that were sensed by pressure transducers and transmitted to the EndoPAT-2000 device. A decrease in the arterial blood volume in the distal fingertip caused a decrease in pulsatile arterial volume change, and was reflected as a decrease in the measured PAT signal and vice versa.

After a baseline recording of 5 minutes on each arm, arterial flow was then interrupted by rapidly occluding blood flow through the brachial artery for 5 minutes using an inflatable cuff. The occlusion pressures were set to $200 \mathrm{~mm}$ $\mathrm{Hg}$ or $60 \mathrm{~mm} \mathrm{Hg}$ plus systolic blood pressure (whichever was higher) in the experimental hand. The PAT signal, which was elicited by the downstream hyperemic response through occlusion of blood flow, was continuously recorded on a computer during the test. Occlusion of pulsatile arterial flow was confirmed by the reduction of the PAT tracing to zero.

After 5 minutes of occlusion, the cuff pressure was rapidly deflated, and postocclusion recording continued for another 5 minutes in both arms. The response to reactive hyperemia, which was induced by occluding the blood vessel, is calculated automatically by the system and a ratio is generated using the postocclusion and preocclusion amplitude values. The index of endothelial function is provided as a reactive hyperemia index (RHI), the post to preocclusion PAT signal ratio in the occluded side, normalized to the control side and further corrected for baseline vascular tone. An RHI score $\leq 1.67$ is abnormal and correlates with endothelial dysfunction, and a score $>1.67$ reflects intact endothelial tone. It was expected that women with a PAT score $\leq 1.67$ were at increased risk of developing preeclampsia. Typical examples of normal and abnormal Endo-PAT traces are shown in - Fig. 1. Patients, whose Endo-PAT could not assess the endothelial function, were excluded from the final analysis.

All endothelial function assessments were performed in the same temperature-controlled exam room. Subjects were followed throughout the gestation until 2 weeks of postpartum. Data regarding demographics and clinical characteristics during pregnancy were assessed from obstetric charts, including clinic visits, emergency visits, and hospitalizations. 

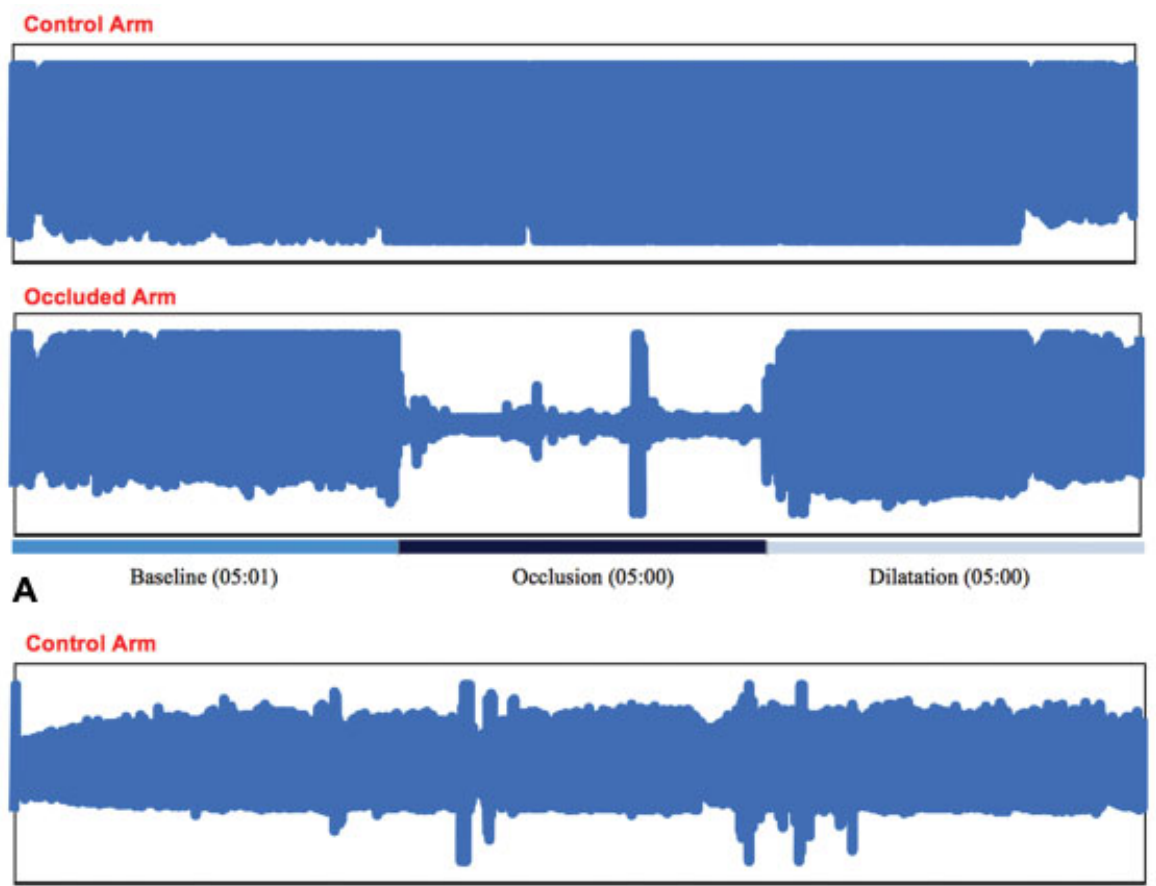

Occluded Arm

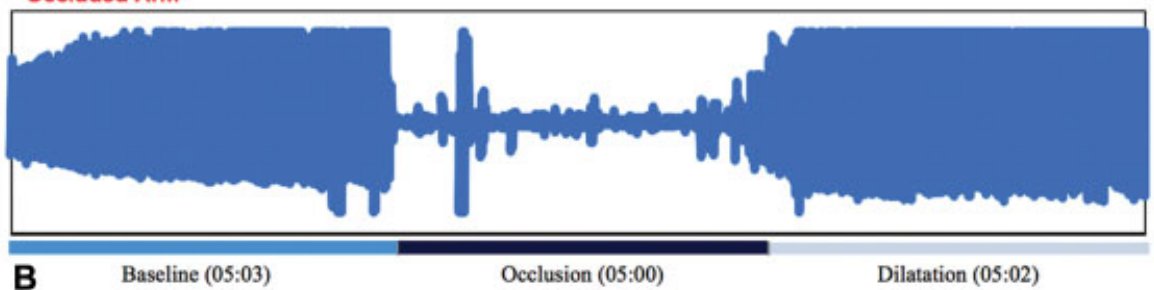

Fig. 1 Normal endothelial function (A) and endothelial dysfunction (B) measured by Endo-PAT. Baseline = preocclusive phase and dilatation $=$ postocclusive phase. The patient with normal endothelial function had an RHI score of 2.48, pulse of 102, and BP was 118/70. The patient with endothelial dysfunction had an RHI score of 1.43, pulse of 98, and BP was 133/62. Signal amplitude in both the preocclusive phase (baseline) and postocclusive phase (dilatation) was lower in the case of endothelial dysfunction (B). BP, blood pressure; PAT, peripheral arterial tone; RHI, Reactive Hyperemia Index.

The primary outcome of preeclampsia was defined as the occurrence of new onset hypertension, defined as blood pressure greater than or equal to $140 \mathrm{~mm} \mathrm{Hg}$ systolic or $90 \mathrm{~mm} \mathrm{Hg}$ diastolic on two occasions at least 4 hours apart after 20 weeks of gestation or greater than or equal to $160 \mathrm{~mm} \mathrm{Hg}$ systolic or $110 \mathrm{~mm} \mathrm{Hg}$ diastolic confirmed within a short interval, in a woman with previously normal blood pressure with proteinuria or other manifestations of preeclampsia. ${ }^{1,2}$ Additionally, proteinuria was defined as greater than or equal to $300 \mathrm{mg}$ per 24 -hour urine collection or protein/creatinine ratio greater than or equal to 0.3 or urine dipstick reading of $1+$. In the absence of proteinuria, preeclampsia was diagnosed as hypertension in association with thrombocytopenia (platelet count less than $100,000 / \mu \mathrm{L}$ ), impaired liver function (elevated blood levels of liver transaminases to twice the normal concentration), new development of renal insufficiency (elevated serum creatinine greater than $1.1 \mathrm{mg} / \mathrm{dL}$ or the doubling of serum creatinine in the absence of other renal disease), pulmonary edema or new-onset cerebral or visual disturbances. ${ }^{1,2}$

\section{Statistical Analysis}

\section{Study Power Analysis}

Women with high-risk factors at 20 weeks of gestation and above were analyzed until delivery. We expected a high degree of sensitivity and specificity from the EndoPAT determination with both being at least $85 \%$ and assumed prevalence of endothelial dysfunction of $45 \%{ }^{10} \mathrm{~A}$ more conservative estimate of $65 \%$ for both required a total sample size of 82 patients for $80 \%$ power with $\alpha=0.05$ and 112 for $90 \%$ power. A larger sample will be required to allow for exploration of additional factors, which may be associated with preeclampsia.

Our independent variable or predictor was the RHI as measured by Endo-PAT. The primary outcome is a diagnosis of preeclampsia as defined above.

\section{Result Analysis}

A receiver operating characteristic ( $\mathrm{ROC}$ ) plot was constructed, predicting preeclampsia from RHI raw score. Logistic regression was used to predict preeclampsia from RHI raw score, 
Table 1 Demographics among women with and without endothelial dysfunction

\begin{tabular}{|c|c|c|c|}
\hline & $\begin{array}{l}\text { Patients with endothelial } \\
\text { dysfunction } \\
n=61\end{array}$ & $\begin{array}{l}\text { Patients without endothelial } \\
\text { dysfunction } \\
n=38\end{array}$ & $p$-Value \\
\hline Maternal age $(\mathrm{y})$ & $31.0 \pm 7.1$ & $32.6 \pm 5.6$ & 0.218 \\
\hline Nulliparity & $10(16.4)$ & $5(13.2)$ & 0.662 \\
\hline Multigestation & $12(19.7)$ & $4(10.5)$ & 0.229 \\
\hline IVF & 7 (11.5) & 0 & 0.03 \\
\hline Body mass index & $32.2 \pm 1.2$ & $31.6 \pm 1.1$ & 0.736 \\
\hline Gestational age & $29.0 \pm 0.6$ & $27.6 \pm 0.8$ & 0.190 \\
\hline History of smoking & $3(4.9)$ & $3(7.9)$ & 0.546 \\
\hline History of preeclampsia & $4(6.6)$ & $6(15.8)$ & 0.138 \\
\hline History of pregestational diabetes & $12(19.7)$ & $10(26.3)$ & 0.439 \\
\hline History of gestational diabetes & $10(16.4)$ & $2(5.3)$ & 0.099 \\
\hline Antidiabetic use & $17(27.9)$ & $9(23.7)$ & 0.645 \\
\hline History of chronic HTN & $11(18.0)$ & $10(26.3)$ & 0.327 \\
\hline Aspirin use & $18(29.5)$ & $13(34.2)$ & 0.624 \\
\hline History of autoimmune disease & $1(1.6)$ & $1(2.6)$ & 0.733 \\
\hline Abnormal uterine artery Doppler & $1(1.6)$ & $1(2.6)$ & 0.733 \\
\hline Abnormal analytes & $6(9.8)$ & $4(10.5)$ & 0.912 \\
\hline
\end{tabular}

Abbreviations: HTN, hypertension; IVF, in vitro fertilization.

Data are $n(\%)$ or mean \pm standard deviation.

body mass index score, gestational age at RHI evaluation, history of preeclampsia, history of pregestational diabetes mellitus, chronic hypertension, and fetal number. The utility of polynomial terms in scored predictors was investigated; a test of interaction between RHI and gestational age was conducted. The Hosmer-Lemeshow test of goodness of fit was applied. The c-statistic (which ranges from 0.5 to 1.0 ) was used as a measure of quality of model fit. About $95 \%$ confidence intervals were computed for area under the ROC plot and for RHI odds ratios (ORs).

\section{Results}

A total of 107 pregnant women were enrolled. Eight of those had undetectable RHI scores (the Endo-PAT could not assess endothelial function), and were excluded from the final analysis.

The demographics among women with and without endothelial dysfunction are summarized in - Table 1. There were no differences seen between women with endothelial dysfunction and women without endothelial dysfunction.

Table 2 Pregnancy outcomes among women with and without endothelial dysfunction

\begin{tabular}{|c|c|c|c|}
\hline & $\begin{array}{l}\text { Patients with endothelial } \\
\text { dysfunction } \\
n=61\end{array}$ & $\begin{array}{l}\text { Patients without endothelial } \\
\text { dysfunction } \\
n=38\end{array}$ & $p$-Value \\
\hline Preeclampsia & $28(17)$ & $16(6)$ & 0.166 \\
\hline Preeclampsia with severe features & $29(5 / 17)$ & $17(1 / 6)$ & 0.541 \\
\hline Gestational age at delivery $\left(\mathrm{wk}^{\mathrm{a}}\right)$ & $37.3 \pm 2.4$ & $36.8 \pm 2.9$ & 0.337 \\
\hline \multicolumn{4}{|l|}{ Mode of delivery } \\
\hline Spontaneous & 36 & 20 & 0.110 \\
\hline Vacuum assisted & 2 & 6 & 0.307 \\
\hline Cesarean & 62 & 74 & 0.245 \\
\hline Birth weight $\left(g^{a}\right)$ & $2,904.2 \pm 709.4$ & $2,861.4 \pm 777.6$ & 0.788 \\
\hline
\end{tabular}

Data are \% (n), unless otherwise stated.

${ }^{a}$ Data are mean \pm standard deviation. 
Delivery characteristics are summarized in - Table 2 . There were no differences in gestational age at delivery, mode of delivery, and birth weight between women with endothelial dysfunction and women without endothelial dysfunction. Among 21 women with a history of chronic hypertension, ten (47.6\%) developed superimposed preeclampsia.

Among the remaining 99 patients, 23 (23\%) eventually developed preeclampsia. When RHI was dichotomized as $>1.67$ versus $\leq 1.67$, total 61 (62\%) patients had abnormal RHI scores and 38 (38\%) had normal RHI scores. Among those with abnormal RHI scores, 17 (28\%) developed preeclampsia and among those with normal RHI scores, 6 (16\%) developed preeclampsia ( $p=0.166$, unadjusted OR: 2.06 , 95\% confidence interval $[\mathrm{CI}]: 0.73-5.81)$. In logistic regression, the RHI term was nonsignificant (adjusted OR: 2.67, 95\% CI: 0.79-9.07, $p=0.116)$. Comparing a model with the RHI predictor to one without, the c-statistic increased from 0.767 to 0.770 .

The bivariate ROC analysis gave no indication of association between RHI score and preeclampsia. In logistic regression, no interaction between RHI score and gestational age was detected. The RHI term was nonsignificant (estimated OR: $0.58,95 \% \mathrm{CI}: 0.15-2.20, p=0.419)$. Comparing a model with the RHI predictor to one without, the c-statistic only increased from to 0.767 to 0.768 .

\section{Discussion}

We found that an abnormal RHI score was not predictive of preeclampsia in women with risk factors for developing preeclampsia. Our findings differ from the prior literature.

Meeme et al tried to use the Endo-PAT 2000 to determine the association between endothelial dysfunction and preeclampsia in pregnant women in rural Africa. They reported that women with preeclampsia show impaired endothelial function (significantly low RHI scores [OR: 1.70, 95\% CI: 1.04-3.61]) compared with normotensive pregnant women (OR: $1.81,95 \% \mathrm{CI}: 1.18-4.62 ; p<0.05) .{ }^{8}$ Although these results seemed to confirm endothelial dysfunction in preeclampsia women, they only evaluated women at the time of diagnosis. They did not study the predictive ability of assessments of endothelial dysfunction.

One study in the United Kingdom similar to ours looked at the correlation between RHI scores and the subsequent development of preeclampsia in women with at least two risk factors. ${ }^{10}$ Those investigators measured RHI scores of 180 patients at 16 and 28 weeks of gestation and followed them until postpartum. They reported no significant differences in RHI scores between women who eventually developed preeclampsia and those who had normotensive pregnancies. Our study confirmed these findings and included women with only one risk factor as well as those with more.

This study has some limitations. Similar to previous studies, we lack data on maternal endothelial health preconception, so it is difficult to know whether the endothelial abnormalities found in some high-risk individuals are consequences of pregnancy or were present before conception. Additionally, our sample size was not large enough to control for multiple risk factors. However, the size was sufficient to evaluate our primary outcome. Finally, our patient population breakdown by ethnicity was 70\% Caucasian, 15\% African American, and 15\% Asian. With this sample size, we were unable to calculate relative risk among minority groups, though the demographic distribution is similar to the distribution in most of the United States.

There are strengths in this study. This was a prospective study of patients with risk factors without preeclampsia allowing us to evaluate the predicative value of this technology. The feasibility of evaluating for endothelial dysfunction in an outpatient setting was demonstrated in this study, and thus this technology could be used in other cohorts to evaluate its utility in screening for patients at increased risk of developing preeclampsia.

\section{Conclusion}

In conclusion, low RHI scores, which are indicative of endothelial dysfunction, did not predict preeclampsia in pregnant women with risk factors for developing preeclampsia. Future studies could recruit a prospective cohort of normotensive pregnant women in the first or early second trimester and follow them until delivery to determine whether RHI measurements could be used as a predictor of preeclampsia in populations without preexisting risk factors of preeclampsia. These studies should also explore whether other biomarkers and/or symptoms can be used in conjunction with the Endo-PAT to more accurately predict the development of preeclampsia.

Conflict of Interest

None declared.

\section{References}

1 Tranquilli AL, Dekker G, Magee L, et al. The classification, diagnosis and management of the hypertensive disorders of pregnancy: a revised statement from the ISSHP. Pregnancy Hypertens 2014;4 (02):97-104

2 ACOG Practice Bulletin No. ACOG practice bulletin no. 202: gestational hypertension and preeclampsia. Obstet Gynecol 2019;133(01):e1-e25

3 Hausvater A, Giannone T, Sandoval YH, et al. The association between preeclampsia and arterial stiffness. J Hypertens 2012;30 (01):17-33

4 Orabona R, Sciatti E, Vizzardi E, et al. Maternal endothelial function and vascular stiffness after HELLP syndrome: a casecontrol study. Ultrasound Obstet Gynecol 2017;50(05): 596-602

5 Orabona R, Sciatti E, Vizzardi E, et al. Endothelial dysfunction and vascular stiffness in women with previous pregnancy complicated by early or late pre-eclampsia. Ultrasound Obstet Gynecol 2017;49(01):116-123

6 Carty DM, Neisius U, Rooney LK, Dominiczak AF, Delles C. Pulse wave analysis for the prediction of preeclampsia. J Hum Hypertens 2014;28(02):98-104

7 Greyling A, van Mil AC, Zock PL, Green DJ, Ghiadoni L, Thijssen DH; TIFN International Working Group on Flow Mediated Dilation. 
Adherence to guidelines strongly improves reproducibility of brachial artery flow-mediated dilation. Atherosclerosis 2016; 248:196-202

8 Meeme A, Buga GAB, Mammen M, Namugowa A. Endothelial dysfunction and arterial stiffness in pre-eclampsia demonstrated by the EndoPAT method. Cardiovasc J Afr 2017;28(01):23-29
9 Carty DM. Preeclampsia; early prediction and long-term consequences. PhD thesis: University of Glasgow; 2012. Available at: http://theses.gla.ac.uk/3124/. Accessed November 11, 2019

10 Carty DM, Anderson LA, Duncan CN, et al. Peripheral arterial tone: assessment of microcirculatory function in pregnancy. J Hypertens 2012;30(01):117-123 